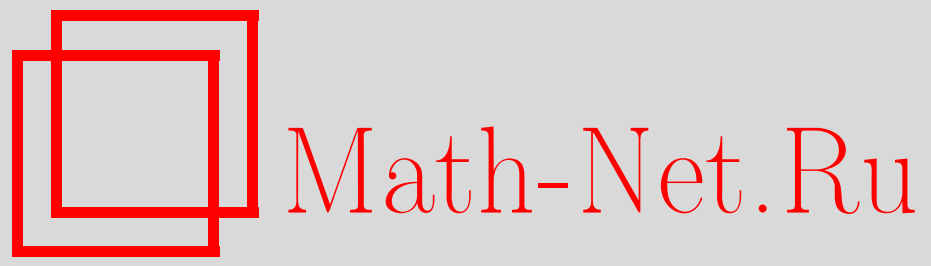

В. А. Васильев, Об одной задаче М. Э. Казаряна, Функи. анализ и его прил., 1999, том 33, Выпуск $3,73-75$

DOI: https: //doi.org/10.4213/faa367

Использование Общероссийского математического портала MathNet.Ru подразумевает, что вы прочитали и согласны с пользовательским соглашением

http://www . mathnet.ru/rus/agreement

Параметры загрузки:

IP: 35.173 .219 .12

26 апреля 2023 г., 10:24:32

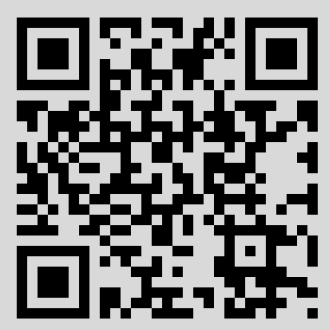




\title{
Об одной задаче М. Э. Казаряна*
}

\author{
(C) 1999. В. А. ВАСИльЕВ
}

Мы доказываем, что группа симметрий ростка функции с нулевой 2-струей и изолированной особенностью не имеет компактных подгрупп положительной размерности.

Обозначим через $\operatorname{Diff}_{0}\left(\mathbb{C}^{n}\right)$ и $\operatorname{Diff}_{0}\left(\mathbb{R}^{n}\right)$ соответственно группы ростков диффеоморфизмов $\left(\mathbb{C}^{n}, 0\right) \rightarrow\left(\mathbb{C}^{n}, 0\right)$ и $\left(\mathbb{R}^{n}, 0\right) \rightarrow\left(\mathbb{R}^{n}, 0\right)$.

Tеорема $1_{\mathbb{C}}$. Допустим, ито $f:\left(\mathbb{C}^{n}, 0\right) \rightarrow(\mathbb{C}, 0)$ - росток голоморфной функции с изолированной особенностью в 0 и его 2 -струя $j^{2} f$ равна 0 . Пусть $G$ - некоторая компактная группа Ли его симметрий, т.е. подгруппа в $\operatorname{Diff}_{0}\left(\mathbb{C}^{n}\right)$, такал, ито $f \circ g \equiv f$ для любого $g \in G$. Тогда $G$ дискретна.

Теорема $1_{\mathbb{R}}$. Допустим, ито $f:\left(\mathbb{R}^{n}, 0\right) \rightarrow(\mathbb{R}, 0)$ - росток $C^{\infty}$-гладкой функиии с конечныл иислом Милнора и его 2-струя $j^{2} f$ равна 0 . Пусть $G \subset$ $\operatorname{Diff}_{0}\left(\mathbb{R}^{n}\right)$ - некоторая компактная группа его симметрий. Тогда $G$ дискретна.

Последнее утверждение было высказано М. Э. Казаряном в качестве гипотезы в связи с его работами по эквивариантной теории гомологий (см. [3] и его же приложение к русскому переводу книги [5]). А именно, согласно этим работам, наша теорема $1_{\mathbb{R}}$ завершает доказательство следующего утверждения:

Группы когомологий введенного в [5] универсального комплекса классов стабильной правой эквивалентности ростков функций изоморфнь (по модулю кручения или с коэффичиентами в поле нулевой характеристики) группе когомологий стабильного лагранжева грассманиана. В частности, все челочисленные лагранжевы характеристические классы задаются как классы, двойственные по Пуанкаре $к$ подходящим ииклам лагранжевых особенностей.

Доказательства. Пусть $G$ - компактная положительномерная группа симметрий вещественной или комплексной функции $f$ с конечным числом Милнора.

Предложение 1 (А. Картан, С. Бохнер, см., например [4]). Голоморфное (соответственно $C^{\infty}$-гладкое) действие компактной группь Ли на комплексном (соответственно вещественном) гладком многообразии вблизи своей неподвижной точки голоморфно (соответственно $C^{\infty}$-) эквивалентно линейному.

Итак, мы можем считать, что наша группа $G$ является подгруппой группы $G L\left(\mathbb{C}^{n}\right)$ (соответственно $\left.G L\left(\mathbb{R}^{n}\right)\right)$. Тогда в силу теоремьи о достаточной cmpye (см., например [2]) она также является группой симметрий некоторой полиномиальной функции с конечным числом Милнора, а именно любого достаточно высокого тейлоровского приближения ростка $f$ в 0 . Поэтому мы можем считать, что $f$ - полином. Если $f$ - вещественная функция, то очевидное продолжение действия группы $G$ в комплексную область сохраняет очевидную комплексификацию полинома $f$; поэтому теорема $1_{\mathbb{R}}$ сводится к теореме $1_{\mathbb{C}}$.

Наконец, мы можем считать, что $G=S^{1}$. Действительно, любая недискретная компактная группа Ли содержит такую подгруппу. Отождествим ее с еди-

* Выполнено при поддержке Миллеровского института фундаментальных научных исследований, INTAS (проект 96-713) и РФФИ (проект 98-01-00555). 
ничной окружностью в $\mathbb{C}^{1}$, так что координата $t$ на $S^{1}$ - это комплексное число, такое, что $|t|=1$.

Из леммы Шура и теоремы Петера-Вейля немедленно следует, что любое представление абелевой компактной группы Ли в $\mathbb{C}^{n}$ распадается в прямую сумму одномерных представлений.

В случае группы $S^{1}$ это означает, что найдутся целые числа $a_{1}, \ldots, a_{n}$, такие, что в подходящих линейных координатах $z_{1}, \ldots, z_{n}$ на $\mathbb{C}^{n}$ это действие имеет следующий вид:

$$
G_{t}:\left(z_{1}, \ldots, z_{n}\right) \mapsto\left(t^{a_{1}} z_{1}, \ldots, t^{a_{n}} z_{n}\right) .
$$

Рассмотрим тейлоровское разложение функции $f$ в этой системе координат: $f=\sum_{\alpha \in \mathbb{Z}_{+}^{n}} f_{\alpha} z^{\alpha}$, где $\alpha=\left(\alpha_{1}, \ldots, \alpha_{n}\right)$ - мультииндексы, $f_{\alpha}$ - коэффициенты, a $z^{\alpha} \equiv z_{1}^{\alpha_{1}} \cdots z_{n}^{\alpha_{n}}$.

Поскольку мы предполагаем, что действие группы $S^{1}$ нетривиально, по меньшей мере одно из чисел $a_{i}$ отлично от нуля. Поэтому все мультииндексы $\alpha$, соответствующие ненулевым коэффициентам $f_{\alpha}$, лежат в гиперплоскости $L(a)$, заданной условием $a_{1} \alpha_{1}+\cdots+a_{n} \alpha_{n}=0$.

Рассмотрим пересечение этой гиперплоскости с симплексом в $\mathbb{R}_{+}^{n}$, выделенным уравнением $\sum \alpha_{i}=3$.

Этот симплекс содержит $n$ меньших симплексов $\Delta_{1}, \ldots, \Delta_{n}$, гомотетичных ему и расположенных при всех его вершинах, а именно, $\Delta_{i}$ - выпуклая оболочка точки $(0, \ldots, 0,3,0, \ldots, 0)$ (3 на $i$-м месте) и $n-1$ точек, получающихся из нее заменой 3 на 2 и одного из нулей на 1.

Теперь теорема $1_{\mathbb{C}}$ вытекает из следующих двух предложений.

Рассмотрим выпуклую оболочку объединения всех точек $\alpha \in \mathbb{Z}^{n}$, таких, что $f_{\alpha} \neq 0$, и начала координат в $\mathbb{Z}^{n}$. Очевидно, эта выпуклая оболочка лежит в плоскости $L(a)$.

ПРЕДЛОЖЕНИЕ 2. Если $f$ имеет изолированную особенность в 0 u $j^{2} f=0$, то эта выпуклая оболочка должна пересекаться со всеми $n$ симплексами $\Delta_{i}$.

ПРЕДЛОЖЕНИЕ 3. Гиперплоскость в $\mathbb{R}^{n}$, проходящая через начало координат, не может пересекать все $n$ симплексов $\Delta_{i}$ одновременно.

ДОКАЗАТЕЛЬСТВо ПРЕДлОЖЕНИЯ 2. Полином $f$ определяет изолированную особенность; поэтому для любого $i$ он содержит моном $f_{\alpha} z^{\alpha}, f_{\alpha} \neq 0$, показатель $\alpha$ которого лежит на расстоянии не более 1 от $i$-й координатной оси (см. например $[1,2])$. Но если $\operatorname{deg} \alpha \geqslant 3$, то отрезок, соединяющий такую точку $\alpha$ и 0 , пересекает симплекс $\Delta_{i}$.

Предложение 3 эквивалентно следующему утверждению: знак определителя любых векторов $v_{1}, \ldots, v_{n}, v_{i} \in \Delta_{i}$, не зависит от выбора этих $n$ векторов.

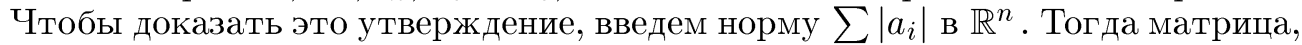
образованная координатными разложениями наших $n$ векторов $v_{i}$, равна $2 I+A$, где $I$ - единичная матрица, а $\|A\| \leqslant 1$. Определитель любой такой матрицы всегда положителен, и наше предложение доказано.

Я очень благодарен В. В. Сергановой и М. Э. Казаряну за полезные беседы.

\section{ЛиТЕРАТУРА}

1. Арнольд В. И. УМН, 29, вып. 2, 9-49 (1974). 2. Арнольд В. И., Варченко А. Н., Гусейн-Заде С. М. Особенности дифференцируемых отображений. Т. 1, Наука, М., 1982. 3. Казарян М. Э. УМН, 50, вып. 4, 45-70 (1995). 4. Кушниренко А. Г. Функц. анализ и его прил., 1, вып. 1, 103-104 (1967). 5. Vassiliev V. A. Lagrange and Legendre 
Characteristic Classes, 2-d ed., Gordon \& Breach Science Publ., New York a.o., 1993. Русский перевод: Васильев В. А., Лагранжевы и лежандровы характеристические классы. Изд-во МЦНМО, 1999.

Математический институт им. В. А. Стеклова РАН

Поступило в редакцию 27 апреля 1999 г.

УДК 517.9

\title{
Об одном представлении для решения Йоста обыкновенных дифференциальных уравнений
}

\author{
(C) 1999. И. М. ГУСЕЙНОВ
}

В 1955 г. Б. Я. Левин [1] ввел «треугольное» представление для решения дифференциального уравнения второго порядка, сохраняющего асимптотику в бесконечности, т.е. для решения Йоста. Это представление играло важную роль при решении обратных задач теории рассеяния (см., например, [2]). Для дифференциальных уравнений высокого порядка с аналитическими коэффициентами подобная задача решена в работах [3-5]. Вопрос о необходимости условия аналитичности коэффициентов изучен в [6].

В настоящей работе получены интегральные представления для решения Йоста дифференциальных уравнений высокого порядка, коэффициенты которых могут быть также и неаналитическими функциями. Для описания свойств ядра представления оказалось естественным использование операторов дробного интегрирования и дробного дифференцирования Римана-Лиувилля

$0<\alpha<1$.

$$
I_{t}^{\alpha} \varphi(x, t)=\frac{1}{\Gamma(\alpha)} \int_{0}^{t}(t-s)^{\alpha-1} \varphi(x, s) d s, \quad D_{t}^{\alpha} \varphi(x, t)=\frac{\partial}{\partial t} I_{t}^{1-\alpha} \varphi(x, t),
$$

Несмотря на нетреугольность этих представлений, свойства ядра, в частности, его связь с коэффициентами уравнения, показывают, что их можно использовать в теории обратных задач.

Рассмотрим дифференциальное уравнение $(n>2)$

$$
y^{(n)}+\sum_{j=0}^{n-2} p_{j}(x) y^{(j)}=\lambda^{n} y, \quad x \in \mathbb{R},
$$

где функции $p_{j}(x)$ принадлежат $C^{j}(\mathbb{R})$ и удовлетворяют условиям

$$
\int_{-\infty}^{\infty}(1+|x|)^{n-1-j+s}\left|p_{j}^{(s)}(x)\right| d x<+\infty, \quad 0 \leqslant s \leqslant j \leqslant n-2 .
$$

Введем следующие обозначения:

$$
\begin{gathered}
q_{j}(t)=\sum_{m=j}^{n-2}(-1)^{m} C_{m}^{j} p_{m}^{(m-j)}(t), \\
(J \psi)(x)=\int_{-\infty}^{x} \psi(t) d t, \quad \sigma(x)=\sum_{j=0}^{n-2}\left(J^{n-j}\left|q_{j}\right|\right)(x) .
\end{gathered}
$$

